Pathologie végétale

\title{
Variabilité biologique du virus de la mosaïque du chou-fleur sur chou-fleur en Bretagne
}

\author{
C. Kerlan et S. Mevel \\ INRA, Station de Pathologie végétale, 35650 Rennes, Le Rheu, France
}

(reçu le 30-5-1988, accepté le 30-9-1988)

Résumé - L'analyse d'échantillons de chou-fleur infectés par le CaMV, prélevés dans les trois zones de production en Bretagne et dans des collections de matériel génétique, met en évidence une variabilité biologique du virus. Les réactions de deux plantes-hôtes, Nicotiana clevelandii Gray et Brassica campestris $L$. Just Right permettent de classer les isolats en quatre groupes et 12 sous-groupes; 11 de ceux-ci sont présents dans le matériel étudié. Une forte proportion de ces isolats $(42 \%)$ induit sur $N$. clevelandii une réaction d'hypersensibilité. L'indexage sur deux populations de choufleur révèle des degrés d'aggressivité différents selon les isolats, mais les réactions ne sont pas uniformes en raison de l'hétérogénéité génétique de ce matériel. A l'opposé, l'hybride B. campestris L. Just Right présente un comportement homogène vis-à-vis d'un isolat donné et constitue une meilleure plante hôte pour mettre en évidence des différences d'aggressivité du CaMV isolé à partir de chou-fleur. Après clonage sur cette plante hôte, les 11 isolats représentant chacun des sous-groupes apparaissent stables.

isolats - indexage - symptomatologie - ELISA

Summary - Biological variability of cauliflower mosaic virus in cauliflower crop in Brittany. Analysis of CaMV infected samples of cauliflower, 38 collected in the fields of the three growing areas in Brittany - a range including summer, autumn and winter crops -, ten in breeding material collections and five standard strains, shows a biological variability of the virus. Symptoms appearing in two host plants, Nicotiana clevelandii Gray and Brassica campestris $L$. Just Right, allow the classification of the isolates in four groups and 12 sub-groups; 11 of them are present in the material tested. A high percentage of these isolates (42\%) induces a hypersensitive reaction in $\mathrm{N}$. clevelandii. Indexing on two cauliflower populations - a locally cultivated variety and one breeding line - shows aggressivity levels varying with the sampling, but due to of the genetic heterogeneity of this material, reactions are not homogeneous with a given isolate. Conversely, we obtain homogeneous reactions on the hybrid B. campestris $L$. Just Right, so this host plant represents a good indicator for revealing aggressivity variations of CaMV isolated from cauliflower. After cloning on this host plant, the 11 isolates selected as representatives of each subgroup appear stable.

isolates - biological indexing - symptomatology - ELISA

\section{Introduction}

Le virus de la mosaïque du chou-fleur (CaMV) (Shepherd, 1981) a une gamme d'hôtes qui ne se restreint pas aux Crucifères, mais c'est chez les espèces cultivées de cette famille qu'il provoque des maladies ayant assez régulièrement un caractère épidémique. Depuis que la relation entre CaMV et étiologie de diverses maladies est connue, ce virus a ainsi été rendu responsable dans le passé d'importantes pertes de rendement en culture de chou-fleur (Brassica oleracea L. var. Botrytis L.) aux USA (Tompkins, 1937), en Grande-Bretagne (Broadbent, 1958) et en France (Spire et André, 1964). Plus récemment, des épidémies sont apparues en Grande-Bretagne sur chou-fleur (Tomlinson et Shepherd, 1978), et sur différents types de choux horticoles dont le chou de Bruxelles $(B$. oleracea $L$. var. gemnifera Zenker) (Tomlinson et Ward, 1981), justifiant pour 
cette dernière espèce la mise en œuvre d'un programme de sélection pour la résistance (Pink et al., 1986). En 1982, le CaMV a été associé à des dommages importants en Géorgie et en Caroline (USA) sur une autre production légumière $(B$. oleracea L. var. acephala D.C.) (Khan et Demski, 1982). Dernièrement, il a été démontré que le CaMV pouvait réduire de façon drastique la production de graines chez le colza oléagineux (Walsh, 1986).

En France, c'est très certainement sur choufleur que le CaMV a la plus forte incidence économique, en raison en premier lieu de l'importance des surfaces cultivées (40 000 ha) et des tonnages récoltés (500 000 t). En Bretagne, région qui fournit les trois quarts de cette production, la maladie de la "mosaïque du chou-fleur» n'est plus aussi grave que dans le passé, mais des foyers loaclisés accompagnés de sensibles pertes de rendement apparaissent chaque année (Kerlan et Mervel, 1987a); de ce fait, elle a été jugée suffisamment préoccupante pour que le CaMV, principal agent pathogène, fasse l'objet d'un programme de recherche d'une méthode de lutte basée sur la résistance génétique de la plante cultivée.

Le préliminaire indispensable à ce travail concerne l'étude de la variabilité du virus avec l'éventuelle mise en évidence de populations virales différant par leur aggressivité sur choufleur. Le présent article décrit les premiers résultats obtenus dans ce domaine à la suite, d'une part, d'une prospection effectuée dans toute la région légumière en Bretagne, et d'autre part, de l'analyse de différents matériels génétiques.

\section{Matériel et Méthodes}

\section{Matériel}

\section{Chou-fleur}

Trente-huit échantillons ont été prélevés dans les trois principales zones de culture en Bretagne (NordFinistère, Côtes-du-Nord et région de Saint-Malo en Illeet-Vilaine), l'échantillonnage prenant également en compte les trois grands types de production, chou-fleur d'été, chou-fleur d'automne et chou-fleur d'hiver-printemps, ainsi que la présence de foyers de maladie grave ou non (Tableau 1). Tous ces prélèvements ont été effectués dans les champs de production.

Dix échantillons concernent du matériel génétique sélectionné par les stations INRA d'Amélioration dess Plantes de Rennes - Le Rhieu et Rennes - Plougoulm (Tableau I).

\section{Virus}

Cinq souches de référence ont été utilisées; la souche Cabb-S (Lebeurier et al., 1978) nous a été fournie par Givord de I'IBMC à Strasbourg; les souches PV 45ATCC- (Pirone et., 1961), PV 147-ATCC- (Shepherd et al., 1970), CM4-184 (Shalla et al., 1980), Cabb.B-J (Delseny et Hull, 1983) nous ont été adressées par Yot de I'INRA-CNRS à Toulouse. Ces souches ont été maintenues en serre sur Brassica campestris L. Just Right.

\section{Plantes tests}

Deux populations de chou-fleur sélectionnées à l'INRA de Rennes nous ont été fournies par Hervé : une population fermière stabilisée FM 60 et une lignée 85 H 115 .

Brassica campestris L. Just Right est un hybride commercial (Atlee Burpee, Co. Warminster, USA). Nicotiana clevelandii Gray est régulièrement multiplié en serre à partir d'un envoi original de Dunez de l'INRA de Bordeaux.

\section{Méthodes}

L'indexage des plantes tests a été réalisé par inoculation mécanique selon la technique classique avec homogénéisation de l'inoculum dans du tampon phosphate à $0,05 \mathrm{M}, \mathrm{pH}=7$, additionné de Dieca à $0,02 \mathrm{M}$. L'incubation a eu lieu en serre hermétique aux pucerons et régulée $\left(18\right.$ à $\left.20^{\circ} \mathrm{C}\right)$ avec une photo-période de $16 \mathrm{~h}$.

Afin d'éliminer une possible influence de l'état physiologique de la plante d'origine, une première transmission a été effectuée sur $B$. campestris $L$. Just Right, à raison de 3 plantes tests par échantillon; ces dernières ont ensuite servi d'inoculum pour une seconde transmission aux quatre groupes de plantes citées ci-dessus ( 5 plantes tests par échantillon).

Les chloroses sur $B$. campestris ont été notées selon une échelle présentant trois degrés d'intensité croissance $(+,++,++t)$; il en a été de même pour l'ensemble des symptômes sur chou-fleur : + : chlorose nervaire; ++ : chlorose nervaire et panachure internervaire; +++ : forte chlorose, gaufrage des feuilles, fort nanisme de la plante. La lecture des symtômes a eu lieu un mois après l'inoculation.

La technique ELISA (Clark et Adams, 1977) a été mise en œuvre pour la détection du CaMV et du virus de la mosaïque du navet (TuMV) avec des réactifs obtenus à partir de sérums préparés au laboratoire et selon un protocole précédemment décrit pour le CaMV (Kerlan et Mevel, 1987b).

\section{Résultats}

Contrôle des prélèvements par la technique ELISA

Tous les échantillons ont répondu positivement au contrôle par la technique ELISA de la présence du CaMV. Deux prélèvements (les nos 27 et 35 ), non 
Tableau I. Origine et principales caractéristiques du matériel analysé. A : souches virales de référence; B : échantillons prélevés en culture; $\mathrm{C}$ : matériel génétique végétal.

\begin{tabular}{ll}
\hline A & \\
1 & IBMC Strasbourg \\
2 & INRA-CNRS Toulouse \\
3 & INRA-CNRS Toulouse \\
4 & INRA-CNRS Toulouse \\
5 & INRA-CNRS Toulouse
\end{tabular}

B

$\begin{array}{ll}\text { Chou-fleur d'été (Ille-et-Vilaine) } \\ 45 & \text { Le Rheu } \\ 46 & \text { Le Rheu } \\ 47 & \text { Le Rheu } \\ 48 & \text { Le Rheu } \\ 49 & \text { Le Rheu }\end{array}$

Chou-fleur d'automne (Ille-et-Vilaine)

$\begin{array}{ll}10 & \text { Le Rheu } \\ 30 & \text { La Rimbaudais } \\ 32 & \text { Le Rheu } \\ 51 & \text { Le Rheu }\end{array}$

Chou-fleur d'automne (Ille-et-Vilaine)

$\begin{array}{ll}53 & \text { Saint-Benoit-de } \\ 54 & \text { Plougoulm } \\ 55 & \text { Saint-Méloir-de } \\ 56 & \text { Cancale } \\ 57 & \text { Saint-Coulomb }\end{array}$

Chou-fleur d'hiver-printermps (Finistère)

$\begin{array}{ll}9 & \text { Saint-Pol-de-Léon } \\ 15 & \text { Plougoulm } \\ 16 & \text { Plougoulm } \\ 17 & \text { Roscoff } \\ 24 & \text { Berven } \\ 25 & \text { Mespaul } \\ 27 & \text { Locquirec } \\ 29 & \text { Plougonven } \\ 31 & \text { Saint-Pol-de-Léon } \\ 35 & \text { Lesneven } \\ \text { Chou-fleur d'hiver-printemps (Côtes-du-Nord) }\end{array}$

Chou-fleur d'hiver-printemps (Côtes-du-Nord)

$\begin{aligned} 6 & \text { Lézardrieux } \\ 7 & \text { Plouguiel } \\ 8 & \text { Plourivo } \\ 11 & \text { Pleumeur-Gautier } \\ 12 & \text { Penvénan } \\ 13 & \text { Coatréven } \\ 18 & \text { Lézardrieux } \\ 21 & \text { Pleumeur-Gautier } \\ 22 & \text { Pleubian } \\ 23 & \text { Pleudaniel } \\ 26 & \text { Plourivo } \\ 28 & \text { Kérity } \\ 33 & \text { Ploubazlanec } \\ 34 & \text { Plouezec }\end{aligned}$

\section{C}

14 INRA-Le Rheu

19 INRA-Plougoulm

20 INRA-Le Rheu

36 INRA-Le Rheu

37 INRA-Le Rheu

38 INRA-Le Rheu

39 INRA-Le Rheu

40 INRA-Le Rheu

41 INRA-Le Rheu

Souche Cabb-S (dérivée d'un isolat d'origine italienne)

Souche PV 45 (souche californienne, ATCC)

Souche PV 147 (Cabb - virus B strain, ATCC)

Souche Cabb. B-Jl (isolat naturel en Grande-Bretagne)

Souche CM4-184 (souche obtenue en laboratoire, dérivant de CM-1841, non transmissible par Aphides)

juil. 1985

août 1985

août 1985

sept. 1985

sept. 1985

déc. 1985

janv. 1985

déc. 1985

déc. 1985

déc. 1987

juin 1987

déc. 1987

déc. 1987

déc. 1987

déc. 1984

juin 1985

juin 1985

déc. 1984

déc. 1984

déc. 1984

déc. 1984

fév. 1984

déc 1984

déc. 1985

janv. 1985

déc. 1984

déc. 1984

janv. 1985

fév. 1985

janv. 1986

fév. 1985

mars 1986

fév. 1986

juin 1986

mars 1985

mars 1986

fév. 1986

mars 1986

déc. 1984

janv. 1984

déc. 1984

déc. 1985

déc. 1985

déc. 1985

déc. 1985

déc. 1985

déc. 1985
Cas isolé en champ d'essais INRA

Cas isolé en champ d'essais INRA

Cas isolé en champ d'essais INRA

Cas isolé en champ d'essais INRA

Cas isolé en champ d'essais INRA

Foyer limité en champ d'essais INRA

Cas isolé en culture

Foyer limité en champ d'essais INRA

Foyer limité en champ d'essais INRA

Foyer limité en culture

Cas isolé en culture

Cas isolé en culture

Cas isolé en culture

Cas isolé en culture

Cas isolé en culture

Porte-graines

Porte-graines

Foyer limité en culture

Cas isolé en culture

Foyer limité en culture

Cas isolé en culture

Foyer grave en culture

Cas isolé en culture

Cas isolé en culture

Foyer grave en culture

Foyer grave en culture

Foyer grave en culture

Cas isolé en culture

Cas isolé en culture

Foyer grave en culture

Foyer grave en culture

Cas isolé en culture

Cas isolé en culture

Porte-graines

Cas isolé en culture

Cas isolé en culture

Cas isolé en culture

Cas isolé en culture

Génotype mâle-stérile Valentino 30 (A)

Génotype mâle-stérile 78-35

Génotype mâle Valentino 30 (B)

Génotype autofertile 7.914.4.7.3.13

Lignée autofertile mâle 7.719.24.13.12.8

Lignée autofertile mâle 7.719.25.13.43

Génotype (décembre) en voie de stérilisation

Géniteur mâle BJF 11.1.17(1)12

Géniteur mâle BJF 11.1.17(1)6 
retenus par la suite, se sont révélés doublement infectés par le CaMV et le TuMV.

Indexage sur $\mathrm{N}$. clevenlandii et $\mathrm{B}$. campestris $L$. Just Right

\section{Symptomatologie}

Sur $N$. clevelandii, pour certains prélèvements, des lésions locales d'abord chlorotiques apparaissent sur les feuilles inoculées 12 à 15 jours après l'inoculation; elles ont un diamètre d'environ $3 \mathrm{~mm}$ et deviennent très rapidement nécrotiques. Aucun symptôme n'apparaît sur les feuilles non inoculées.

Sur B. campestris L. Just Right, des taches chlorotiques ponctuelles ( 1 à $2 \mathrm{~mm}$ de diamètre) apparaissent sur les feuilles inoculées, et parfois sur les feuilles immédiatement supérieures, 10 à 12 jours après l'inoculation; ce type de symptôme a été obtenu quel que soit l'échantillon indexé; les feuilles non inoculées présentent différentes formes de chloroses et des déformations d'intensité variable; pour certains isolements, des nécroses apparaissent sur les feuilles du coeur de la plante, la croissance de celle-ci étant alors fortement affectée.

\section{Résultats d'analyse des échantillons}

Les résultats figurent au Tableau II, les échantillons étant regroupés par type de matériel : souches de référence (Tableau IIA), chou-fleur d'été et chou-fleur d'automne (Tableau IIB), choufleur d'hiver-printemps (Tableau IIC), matériel génétique (Tableau IID).

\section{Essai de classification des isolements}

Trois types de réactions induites sur ces deux plantes hôtes permettent de classer les 53 isolements en quatre grands groupes et 12 sousgroupes selon le modèle suivant :

- Groupe 1 : lésions locales sur N. clevelandii; nécroses sur $B$. campestris

- sous-groupe IA : fortes chloroses sur B. campestris, 12 isolats : nos 8.10.14.18.24.26.28.31.37. 39.49.54,

- sous-groupe IB : chloroses d'intensité moyenne sur $B$. campestris, 4 isolats : nos 4.22.34.36,

sous-groupe IC : faibles chloroses sur $B$. campestris, 1 isolat : $n^{\circ} 20$;

- Groupe II : lésions locales sur N. clevelandii; pas de nécroses sur $B$. campestris

- sous-groupe IIA : fortes chloroses sur $B$. campestris, 3 isolats : nos 9.21 .55 ,
- sous-groupe IIB : chloroses d'intensité moyenne sur $B$. campestris, 1 isolat : $n^{\circ} 30$,

- sous-groupe IIC : faibles chloroses sur $B$. campestris, aucun isolat;

- Groupe III : pas de lésion locale sur N. clevelandii; nécroses sur $B$. campestris

- sous-groupe IIIA : fortes chloroses sur $B$. campestris, 12 isolats : nos 6.15.16.25.29.40.42.45.47.48.51.53,

- sous-groupe IIIB : chloroses d'intensité moyenne sur $B$. campestris, 5 isolats : nos 7.13.19.38.46,

- sous-groupe IIIC : faibles chloroses sur $B$. campestris, 1 isolat : $n^{\circ} 12$;

- Groupe IV : pas de lésion locale sur N. clevelandii; pas de nécrose sur $B$. campestris

- sous-groupe IVA : fortes chloroses sur $B$. campestris, 4 isolats : nos 3.32.33.56,

- sous-groupe IVB : chloroses d'intensité moyenne sur $B$. campestris, 4 isolats : nos 1.2.17.41,

- sous-groupe IVC : faibles chloroses sur $B$. campestris, 4 isolats : nos 5.11.23.57.

Indexage sur chou-fleur

Afin de vérifier la correlation entre intensité des symptômes sur $B$. campestris $L$. Just Right et sur chou-fleur, le comportement de tous les isolements a été étudié sur 2 populations de chou-fleur sélectionnées arbitrairement : une population fermière stabilisée (FM 60) et une lignée $F 1(85 \mathrm{H}$ 115). On observe pour chaque lot de plantes inoculées une hétérogénéité de réaction liée à l'hétérogénéité génétique de la population de choufleur, y compris chez la lignée $85 \mathrm{H} \mathrm{115}$; la note traduisant l'intensité des symptômes rapportée dans le Tableau III représente donc une valeur moyenne établie à partir des cinq plantes tests.

Cinq échantillons se caractérisent par des symptômes typiquement faibles sur l'une et l'autre population de chou-fleur dont les souches de référence 3,4 et 5 ainsi que les échantillons 12 (choufleur d'hiver dans les Côtes-du-Nord) et 57 (choufleur d'automne en llle-et-Vilaine). Parmi les souches de référence, seule PV 45 est d'intensité moyenne, alors que la souche Cabb-S n'atteint ce niveau que sur la lignée $85 \mathrm{H} 115$.

Les sous-groupes les plus homogènes sont IA, IIA, IIIA et IIIB; à l'opposé, le sous-groupe IB avec trois niveaux d'intensité des symptômes, et à un degré moindre les sous-groupes IVA et IVB, sont 
Tableau II. Résultats de l'indexage sur $N$. clevelandii et $B$. campestris L. Just Right. A : souches de référence; B : choufleur d'été et chou-fleur d'automne; $C$ : chou-fleur d'hiver-printemps; $D$ : matériel génétique.

\section{Souche}

N. clevelandii

nécroses
Réactions induites sur

B. campestris cv. Just Right

nécroses

-
-
+
+

$++$

$++$

$++$

$+$

B

Chou-fleur d'été

45

46

47

48

Chou-fleur d'automne

10
30

32

51

53

54

55

57

C

\section{Finistère}

9

15

16
17

17
24

24
25

29

31 Côtes-du-Nord

6

7

8

11

12

13
18

21

22

23

26

28

33

34

D

Matériel génétique

14

19

20

36

37

38

39

40

41

42

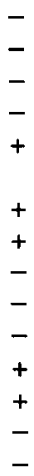

$+++$

$++$

$+++$

$+++$

$++t$

$+++$

$+t$

$+++$

$+++$

$+++$

$+++$

$+++$

$++$

$+$

$+++$

$+++$

$+++$

$+$

$+++$

$+++$

$++$

$+++$

$++$

$++$

$+++$

$+$

$+++$

$+++$

$++$

$+$

$+++$

$+++$

$++$

$++$

* Chloroses notées selon trois degrés d'intensité croissante $(+,++,+++)$.

$+++$

$++$

$+$

$++$

$++$

$+++$

$+++$

$++$

\section{$\operatorname{roses}^{*}$}

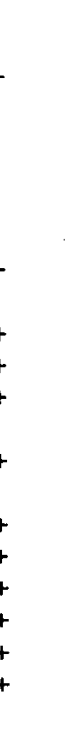


Tableau III. Résultats de l'indexage sur deux populations de chou-fleur.

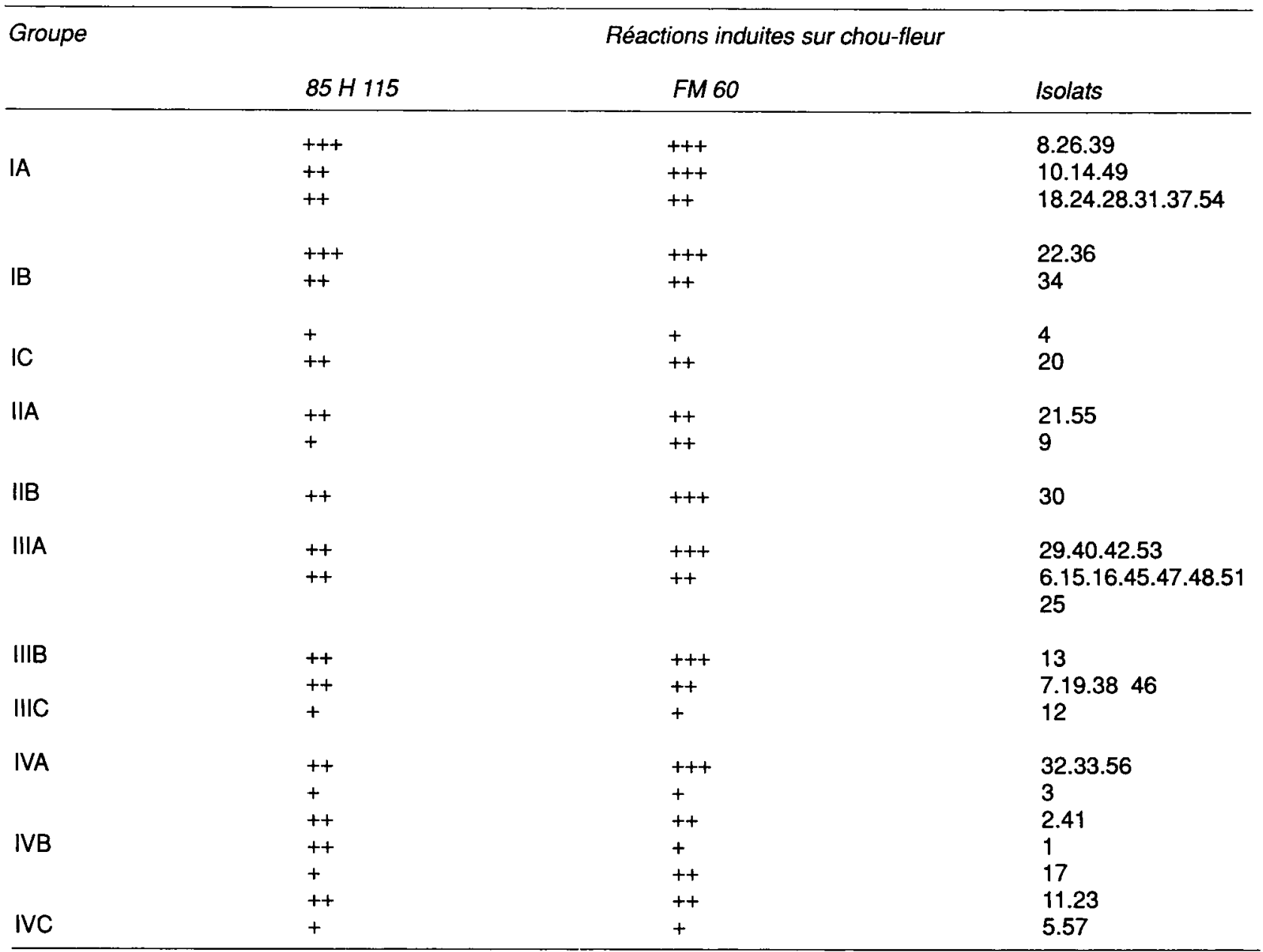

+Chlorose nervaire; ${ }^{++}$chlorose nervaire et panachure internervaire, faible réduction de croissance de la plante; ${ }^{+++f o r t e ~ c h l o r o s e, ~ g a u-~}$ frage des feuilles, fort nanisme de la plante.

hétérogènes. II n'y a donc pas de corrélation entre intensité des symptômes sur $B$. campestris et sur chou-fleur, même si globalement les représentants des sous-groupes $A$ donnent plus de symptômes forts que ceux des sous-groupes $C$.

Toutes les plantes tests ont été contrôlées en ELISA pour la présence de CaMV et ont toutes réagi positivement; les valeurs de densités optiques variaient entre 0,65 et 1,73 et n'étaient pas correlées avec le degré d'intensité des symptômes.

\section{Sélection et clonage d'isolats}

Onze isolats (nos 26.22.20.21.30.29.7.12.32.17.23) ont été sélectionnés en tant que représerltants de chacun des sous-groupes trouvés. Ils ont été clonés par transferts répétés de taches chlorotiques individualisées de $B$. campestris à $B$. cam- pestris en partant de plantes inoculées à un stade très jeune (trois à quatre feuilles). Les essais de clonage à partir de $N$. clevelandii pour les isolats induisant des lésions locales sur cette espèce se sont révélés infructueux. Après clonage, chaque isolat sélectionné a été rétroinoculé sur ces deux espèces indicatrices, B. campestris L. Just Right et $N$. clevelandii, où il a induit une réaction de même type et de même intensité que l'isolement d'origine; aucun de ceux-ci ne renfermait donc un mélange d'isolats.

\section{Discussion}

L'étude de la variabilité du CaMV a été réalisée dans la perspective de travaux ultérieurs sur la résistance génétique du chou-fleur à ce virus. La première tâche résidait dans le choix d'une ou de 
plantes hôtes susceptibles de mettre en évidence des niveaux d'aggressivité différents. Les études antérieures font état, outre le chou-fleur, de 3 plantes différenciatrices : $B$. pekinensis, le navet (Broadbent et Tinsley, 1953; Broadbent, 1958) et B. campestris cv. Just Right (Tomlinson et Shepherd, 1978). Cependant le type exact de navet (variété ou sous-espèce) n'est pas précisé par les auteurs qui le mentionnent. D'autre part des expériences préliminaires d'indexage sur $B$. pekinensis nous ont montré l'hétérogénéité des réactions à l'intérieur d'un lot de plantes inoculées par le même isolat. Par contre, B. campestris cv. Just Right est un hybride qui réagit de façon très homogène et aisément reproductible pour les trois types de symptômes : présence ou absence de lésions locales chlorotiques, intensité des chloroses, extériorisation ou non de nécroses systémiques. La difficulté du choix a priori de variétés standard de chou-fleur généralement encore peu stabilisées génétiquement, le temps d'indexage plus court et une plus grande facilité d'inoculation de $B$. campestris cv. Just Right constituent des arguments supplémentaires pour retenir cet hybride en tant que plante indicatrice du niveau d'aggressivité de l'isolat viral.

A la différence de Broadbent (1958), nous n'obtenons pas une exacte corrélation entre intensité des symptômes sur $B$. campestris cv. Just Right et sur chou-fleur, mais la population fermière et la lignée de chou-fleur expérimentées présentent elles-mêmes des sensibilités différentes et ont chacune un comportement hétérogène. Les trois niveaux de sévérité que nous distinguons sur cet hybride commercial sont analogues à ceux décrits par Tomlinson et Shepherd (1978) sur cette même plante hôte; cependant, à la différence de ces auteurs, CM4-184 nous est apparue comme une souche typiquement faible, avec des symtomes ne dépassant pas le stade de chlorose nervaire; cette même souche CM4-184 est également considérée comme relativement faible par Shepherd (1981).

Par ailleurs, la présence systématique de lésions locales chlorotiques sur les feuilles inoculées de $B$. campestris $\mathrm{cv}$. Just Right constitue une autre caractéristique de nos isolements. En effet, Shepherd (1981) distingue deux catégories de souches, selon qu'elles induisent ou non ce type de réaction sur cette plante hôte; dans des conditions analogues à celles de Shepherd, nous obtenons régulièrement des lésions locales dont seul le nombre varie en fonction de l'isolat. Cette propriété nous a permis de résoudre les problèmes de clonage déjà rencontrés par Tomlinson et Shepherd (1978) en utilisant la voie biologique plus simple et pour nous plus rapide que le clonage moléculaire (Schoelz et al., 1986)

Le CaMV a été longtemps considéré comme inféodé aux Crucifères et n'induisant que des réactions systémiques. Hills et Campbell (1968) font état pour la première fois d'une souche capable d'infecter $N$. clevelandii, alors que Lung et Pirone (1972) découvrent que Datura stramonium est un hôte à lésions locales pour certains isolats. Plus récemment, des souches inhabituelles (W 260, D4), se multipliant systématiquement sur plusieurs Solanées, ont été isolées aux USA (Gracia et Shepherd, 1985; Schoelz et al., 1986). Contrairement à cette souche D4 et à celle de Hills et Campbell, tous nos isolats infectant $N$. clevelandii induisent sur cette plante une réaction d'hypersensibilité stricte, la présence de virus n'étant pas décelable dans les feuilles non inoculées. Le grand nombre d'isolats trouvés en Bretagne ( 20 sur 48 , soit $42 \%$ ) présentant ce type de réactions constitue l'originalité majeure de ce travail et montre que la variabilité biologique de ce virus n'a sans doute pas encore été suffisamment étudiée. Une caractérisation complémentaire de nos isolats sur les espèces de Solanées permettant de distinguer les trois types de souches décrits par Schoelz et Shepherd (1988) est actuellement en cours.

La répartition des isolats en quatre groupes résulte de la prise en compte de deux critères assurant une différenciation particulièrement nette et reproductible. La distinction en 12 sous-groupes permet de sélectionner une gamme suffisamment étendue d'isolats que nous pensons représentatifs de l'ensemble de la population virale sur choufleur en Bretagne, compte tenu de l'importance de la prospection réalisée et de la diversité de l'échantillonnage. A l'exception dans une certaine mesure des souches de référence (groupe IV dominant) et du chou-fleur d'été (groupe III dominant), il n'y a pas de relation stricte entre groupes et origine géographique, type de matériel ou foyer de maladie. Sur chou-fleur, les souches de référence sont généralement faibles, alors que les isolats provenant des foyers de maladie grave sont plutôt forts. L'étude du comportement d'une large gamme de populations de chou-fleur (sélections fermières, lignées et hybrides) vis-à-vis des isolats sélectionnés va maintenant être entreprise.

\section{Références}

Broadbent L. (1958) Investigation of Virus Diseases of Brassica Crops. Cambridge University Press, p. 94 
Broadbent L. \& Tinsley T.W. (1953) Symptoms of cauliflower mosaic and cabbage black ringspot in cauliflower. Plant Pathol. 2, 88-92

Clark M. F. \& Adams A.N. (1977) Characteristics of the microplate method of enzyme-linked immunosorbent assay for the detection of plant viruses. J. Gen. Virol. 34, 475-483

Delseny M. \& Hull R. (1983) Isolation and characterization of faithful and altered clones of the genomes of cauliflower mosaic virus isolates Cabb B-JI, CM4-184, and Bari I. Plasmid 9, 31-41

Gracia O. \& Shepherd R.J. (1985) Cauliflower mosaic virus in the nucleus of Nicotiana. Virology 146, 141-145

Hills G.J. \& Campbell R.N. (1968) Morphology of broccoli necrotic yellows virus. J. Ultrastr. Res. 24, 134-144

Kerlan C. \& Mevel S. (1987a) Situation des viroses du chou-fleur en Bretagne. C.R. Acad. Agr. Fr., 73, 103116

Kerlan C. \& Mevel S. (1987b) Pratical use of ELISA to detect cauliflower mosaic virus in Cauliflower. Eur. Mediterr. Plant Prot. Organ. Bull. 17, 125-130

Khan M.A. \& Demski J.W. (1982) Identification of Turnip mosaic and cauliflower mosaic viruses naturally infecting collards. Plant. Dis. 66, 253-256

Lebeurier G., Whitechurch O., Lesot A. \& Hirth L. (1978) Physical map of DNA from a new cauliflower mosaic infection. Virology 37, 506-508

Lung M.C.Y. \& Pirone T.P. (1972) Datura stramonium, a local lesion host for certain isolates of cauliflower mosaic virus. Phytopathology 62, 1473-1474

Pink D.A.C., Sutherland R.A. \& Walkey D.G.A. (1986) Genetic analysis of resistance in Brussels sprout to cau- liflower mosaic and turnip mosaic viruses. Ann. Appl. Biol. 109, 199-208

Pirone T.P., Pound G.S. \& Shepherd R.J. (1961) Properties and serology of purified cauliflower mosaic virus. Phytopathology 51, 541-546

Schoelz J.E., Shepherd R.J. \& Richins R.D. (1986) Properties of an unusual strain of cauliflower mosaic virus. Phytopathology 76, 451-454

Schoelz J.E. \& Shepherd R.J. (1988) Host range control of cauliflower mosaic virus. Virology 162, 30-37

Shalla T.A., Shepherd R.J. \& Peterson L.J. (1980) Comparative cytology of nine isolates of cauliflower mosaic virus. Virology 102, 381-388

Shepherd R.J. (1981) Cauliflower mosaic virus. CMI/AAB. Desc. Plant Virus, 243, 1, 6

Shepherd R.J., Bruening G.E. \& Wakeman R.J. (1970) Double-Stranded DNA from cauliflower mosaic virus. Virology 41, 339-347

Spire D. \& Andre M. (1984) Le virus de la mosaïque du chou-fleur en Bretagne. Méthodes de lutte à envisager. C.R. Acad. Agr. Fr., 846-841

Tomlinson J.A. \& Shepherd R.J. (1978) Studies on mutagenesis and cross protection of cauliflower mosaic virus. Ann. Appl. Biol. 90, 223-231

Tomlinson J.A. \& Ward C.M. (1981) The reactions of some Brussels sprout F1 hybrids and inbreds to cauliflower mosaic and turnip mosaic viruses. Ann. Appl. Biol. 97, 205-212

Tompkins C.M. (1937) A transmissible mosaic disease of cauliflower. J. Agr. Res. 55, 33-46

Walsh J.A. (1986) Virus infection of oilseed rape, a potential threat to vegetable crops. NVRS Wellesbourne Report 1986 